\title{
Article \\ Hybrid Nonlinear State-Space Modeling Approach for a Dual Armature Generator
}

\author{
Thilina Fernando ${ }^{1}$, Qin Zhou ${ }^{2}$ and Hector M. Gutierrez ${ }^{3, *(D)}$ \\ 1 Hitachi Automotive Systems, Farmington Hills MI 48335, USA; thilinajf@gmail.com \\ 2 School of Electrical Engineering, Shanghai Dianji University, Shanghai 201306, China; zqforyou@outlook.com \\ 3 Department of Mechanical Engineering, Florida Institute of Technology; Melbourne, FL 32901, USA \\ * Correspondence: hgutier@fit.edu; Tel.: +1-321-674-7321
}

Citation: Fernando, T.; Zhou, Q.;

Gutierrez, H.M. Hybrid Nonlinear State-Space Modeling Approach for a Dual Armature Generator. Electronics 2021, 10, 1401. https://doi.org/ 10.3390/electronics10121401

Academic Editors: Albert Smalcerz and Marcin Blachnik

Received: 9 May 2021

Accepted: 7 June 2021

Published: 10 June 2021

Publisher's Note: MDPI stays neutral with regard to jurisdictional claims in published maps and institutional affiliations.

Copyright: (c) 2021 by the authors. Licensee MDPI, Basel, Switzerland. This article is an open access article distributed under the terms and conditions of the Creative Commons Attribution (CC BY) license (https:/ / creativecommons.org/licenses/by/ $4.0 /)$.

\begin{abstract}
A novel hybrid state space modeling approach for a dual armature generator is presented. The model uses finite element analysis to obtain the rotor angle-dependent parameters, namely the flux linkage, stator inductances and cogging torque, which are then incorporated into the dynamic equations of the hybrid model using Fourier representations of the physical parameters as function of the rotor angle. The proposed hybrid model addresses many of the limitations of the classical modeling approach, which uses analytical expressions to obtain flux linkage and stator inductances based on significant simplifications. The proposed model is verified experimentally by simulating the dynamics of a three-phase permanent magnet generator. Predictions based on the new hybrid model are compared to both experimental measurements and predictions based on the classic modeling approach. It is shown that the proposed hybrid model simulates the dynamics of the demonstration machine to within $7 \%$ peak error in the stator current and provides a $13 \%$ improvement to the prediction of the rotor velocity compared with the classic model.
\end{abstract}

Keywords: finite element analysis; flux linkage; stator inductance; cogging torque; dual armature generator

\section{Introduction}

In a conventional permanent magnet synchronous machine, the permanent magnets are fixed to the rotor, and the rotor is surrounded by the armature coils. Flux generated by the side of the permanent magnets not facing the stator is unused. The dual armature concept takes advantage of the unused flux by adding a second set of armature coils which links this previously unused flux, offering advantages such as improved efficiency, a compact size and higher power density. Dual armature generators are becoming more popular and are finding new applications [1,2].

Chai, Cui and Cheng [3] performed a study of the dual armature concept and investigated an 8-pole, 24-slot machine. Chai et al. [4] focused on the torque density of a dual armature motor, and their objective was to optimize the design for the maximum torque. Norhisam, Norafiza and Sia [2] presented a 12-pole, single-phase dual armature generator designed to operate at 8000 RPM, with their focus being to find the ideal overall length of the machine as well as the ideal number of slots for the maximum power output. Zhang et al. [5] outlined the design and analysis of a 22-pole, 3-phase dual armature generator for direct driven wind power applications and used time-stepping finite element analysis (FEA) in their design.

During the design of a dual-armature concept, the limitations of the classical modeling approach for electrical machines becomes quickly apparent. This paper presents a novel hybrid dynamic model that incorporates finite element analysis to obtain geometrydependent motor parameters, which are then incorporated into a hybrid nonlinear state space model of a permanent magnet electrical machine. 


\subsection{Technical Background}

\subsubsection{Dynamic Model of a Permanent Magnet Synchronous Generator}

The standard model [6] of a permanent magnet synchronous generator is the state space description in a rotor-oriented $d, q$ coordinate system. As the influence of the direct axis saturation can be easily measured, at least for an electrically excited synchronous generator, the authors of [7-9] used a unidirectional model. Their suggestion was that the saturation changed equally in both the $\mathrm{d}$ and $\mathrm{q}$ directions. Their models were based on two parameter approaches which have been further developed to an intermediate axis model [10,11].

Lyshevski [12] outlined a dynamic model for a permanent magnet synchronous generator in the time domain, which served as a starting point for the model proposed in this paper. The model incorporates both electrical and mechanical components, which are derived from Kirchhoff's and Newton's laws, respectively:

$$
\begin{gathered}
\vec{U}=-R_{s} \vec{i}+\frac{d \vec{\psi}}{d t} \\
T_{i n}-T_{e}-B_{m} \omega_{m}=J \frac{d \omega_{m}}{d t}
\end{gathered}
$$

where $U$ is the vector of the stator voltages $(3 \times 1), R_{S}$ is the $3 \times 3$ matrix of the stator resistances, $i$ is the $3 \times 1$ vector of the stator currents (state variable), $\omega_{m}$ is the rotor velocity (state variable), $\theta$ is the rotor angle (state variable), $\psi$ is the flux linkage, $J$ is the rotor moment of inertia, $B_{m}$ is the viscous damping of the rotor, $T_{e}$ is the electromagnetic reaction torque and $T_{i n}$ is the input torque applied by the prime mover. The following section describes the elements of this model.

Flux Linkage. The flux linked to the stator coils $(\psi)$ by the permanent magnets is the most important performance parameter in a permanent magnet (PM) machine, and it is given by

$$
\psi=L_{s} \vec{i}+\psi_{m}\left[\begin{array}{c}
\sin (\theta) \\
\sin \left(\theta-\frac{2}{3} \pi\right) \\
\sin \left(\theta+\frac{2}{3} \pi\right)
\end{array}\right]
$$

where $L_{S}$ is the inductance matrix, which has the following form:

$$
L_{s}=\left[\begin{array}{lll}
L_{a a} & L_{a b} & L_{a c} \\
L_{a b} & L_{b b} & L_{b c} \\
L_{a c} & L_{b c} & L_{c c}
\end{array}\right]
$$

The diagonal elements of $L_{s}$ are the stator self inductances, and the off-diagonal elements are the mutual inductances between the stator phases. The matrix is symmetrical about its diagonal. The stator self and mutual inductances are functions of the rotor angle, but in the classical model, they are assumed to be constant. For the standard model, $L_{s}$ is defined as

$$
L_{s}=\left[\begin{array}{ccc}
L_{l s}+L_{m} & -\frac{1}{2} L_{m} & -\frac{1}{2} L_{m} \\
-\frac{1}{2} L_{m} & L_{l s}+L_{m} & -\frac{1}{2} L_{m} \\
-\frac{1}{2} L_{m} & -\frac{1}{2} L_{m} & L_{l s}+L_{m}
\end{array}\right]
$$

where $L_{l s}$ and $L_{m}$ are the quadrature and direct axis inductances, respectively, which for a permanent magnet synchronous machine, assumed to be a round rotor machine, are constant [12]. The inductances are calculated analytically using the following expressions [12,13]:

$$
L_{s}=\frac{\mu N^{2} A}{L} \quad L_{m}=\frac{2 N^{2}}{3 R}
$$

where: $L_{S}$ is the stator self-inductance, $N$ is the number of turns, $A$ is the coil area, $L$ is the coil length, $\mu$ is the magnetic permeability of the core material, $R$ is the reluctance of the magnetic circuit. $\psi_{m}$ is the maximum flux linked to the stator by the permanent magnets 
and $\theta$ is the electrical angle. The angular variation of the flux is assumed to be sinusoidal. While this approximation is acceptable in real machines, the flux linkage waveform is not perfectly sinusoidal:

$$
\psi=\psi_{m}\left[\begin{array}{c}
\sin (\theta) \\
\sin \left(\theta-\frac{2}{3} \pi\right) \\
\sin \left(\theta+\frac{2}{3} \pi\right)
\end{array}\right]
$$

where $\psi_{m}$ is the maximum flux linked to the stator by the permanent magnets, which can be calculated analytically using the procedure below [13]. The operating line for the magnetic circuit is first determined using magnetic circuit analysis as follows:

$$
2 H_{m} l_{m}=-\left[\frac{l_{s} A_{m}}{\mu_{s} A_{s}}+\frac{l_{r} A_{m}}{\mu_{r} A_{r}}+\frac{l_{a} A_{m}}{\mu_{a} A_{a}}\right] B_{r}
$$

where $l_{s}, l_{r}, l_{a}$ and $L_{m}$ are the magnetic circuit lengths for the stator, rotor, air gap and magnet, respectively, $\mu_{s}, \mu_{r}$ and $\mu_{a}$ are the magnetic permeability of the stator, rotor and air, respectively, $A_{m}$ is the cross-section area of the permanent magnet, $A_{s}, A_{r}$ and $A_{a}$ are the areas of the magnetic circuits for the stator, rotor and air gap, respectively, $H_{m}$ is the magnetic coercivity and $B_{r}$ is the magnetic remanence. The above equation applies to the operating line for the magnetic circuit in question. The operating point is the point where the operating line intersects the magnetization curve of the permanent magnet [13].

Electromagnetic Torque. $T_{e}$ is the torque generated by the back electromotive force during operation. This torque acts as the reaction force to the torque input by the prime mover. In the steady state, $T_{e}=T_{i n}$, and the system operates at a constant angular velocity. $T_{e}$ is derived using co-energy and is a function of the electrical angle $\theta$ [12]:

$$
T_{e}=\frac{P}{2} \frac{\partial W_{c}}{\partial \theta}
$$

where $P$ is the number of poles in the machine and $W_{c}$ is the co-energy, which is derived as follows:

$$
W_{c}=\frac{1}{2} \vec{i}^{T} L_{s} \vec{i}+\vec{i}^{T} \psi_{m}+W_{m}
$$

where $L_{s}$ and $\psi_{m}$ are the same as described above and $W_{m}$ is the energy stored in the permanent magnets, which is considered constant [14]. Co-energy is the area under the flux linkage versus the current plot, and the energy stored by the permanent magnets is derived from the magnets' B-H curve. A typical B-H curve for a permanent magnet showing energy stored in its magnetic field is seen in Figure 1 [20]. For the electromagnetic torque expression, the partial derivative of the co-energy with respect to the electrical angle is needed. The classical model assumes $L_{S}$ is constant, so the partial derivative is reduced to

$$
\frac{\partial W_{c}}{\partial \theta}=\overrightarrow{i^{T}} \psi_{m}\left[\begin{array}{c}
\cos (\theta) \\
\cos \left(\theta-\frac{2}{3}\right) \\
\cos \left(\theta+\frac{2}{3}\right)
\end{array}\right]
$$




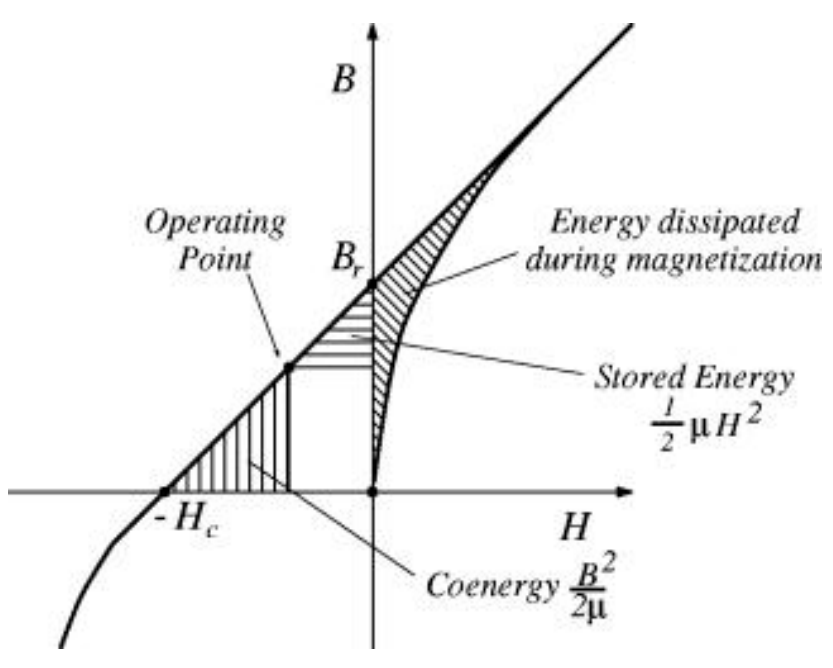

Figure 1. Energy stored in a permanent magnet [20]. B: magnetic field density, $H$ : magnetic field intensity, $B_{r}$ : magnetic remanence; $\mu$ : permittivity; $H_{c}$ : magnetic coercivity.

Cogging Torque. Cogging torque is a phenomenon inherent to all permanent magnet electrical machines, and it is caused by the PMs seeking a configuration that minimizes magnetic reluctance [15]. Cogging torque depends on the rotor angle and is periodic. Cogging torque is a parasitic effect, present even when the machine is not in operation, and it does not contribute to the overall torque. Cogging torque is a major design issue in PM machines; it affects start-up, causes torque ripple and introduces vibrations [15]. Several design strategies can be adopted to mitigate cogging torque, the most common being to skew the armature slots relative to the PMs by one slot pitch. This has the effect of creating an overall constant magnetic reluctance circuit, presenting the magnets with an overall constant reluctance path. Cogging torque can also be mitigated by the use of fractional pitch windings, fractional slot designs [16] and motor control strategies [17]. When modeling electrical machines, cogging torque is treated as an external torque input in both the classical and hybrid models. Figure 2 shows the effects of cogging torque in the demonstration machine (shown in Figures 3 and 4), simulated based on the classical model with and without cogging torque. The bottom plots are zoomed in.
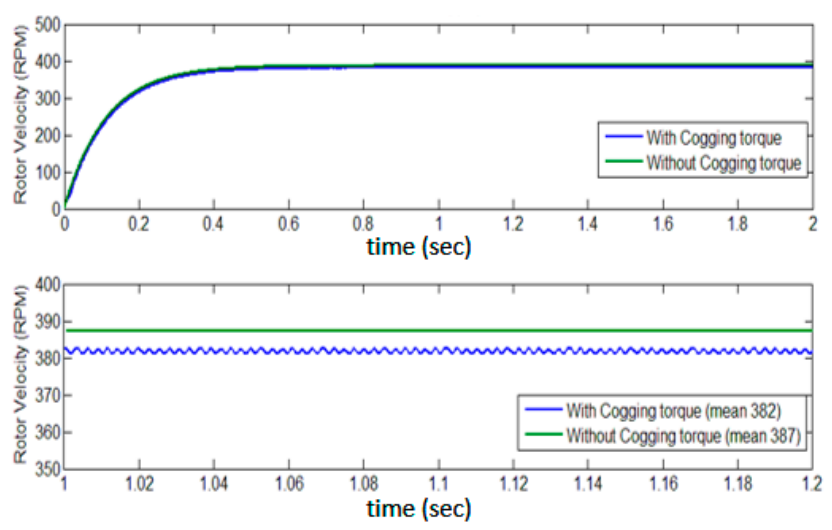

(a)
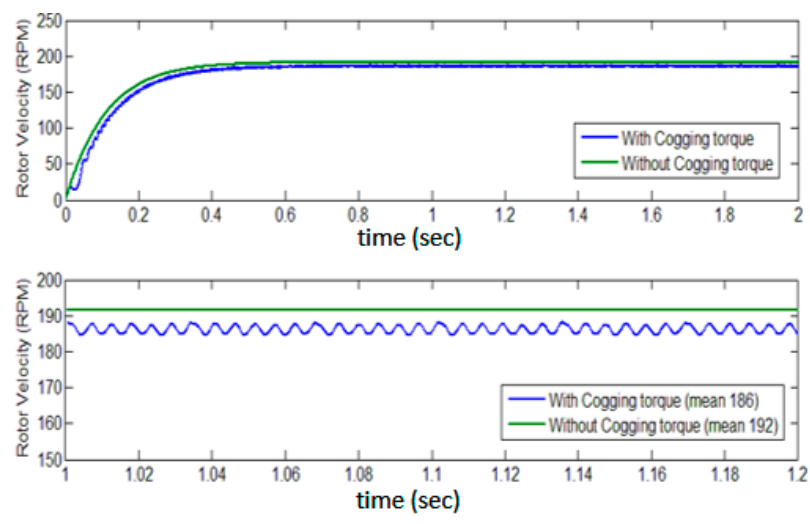

(b)

Figure 2. The effect of cogging torque (a) on a machine rotating at high speed and (b) a machine rotating at low speed. 


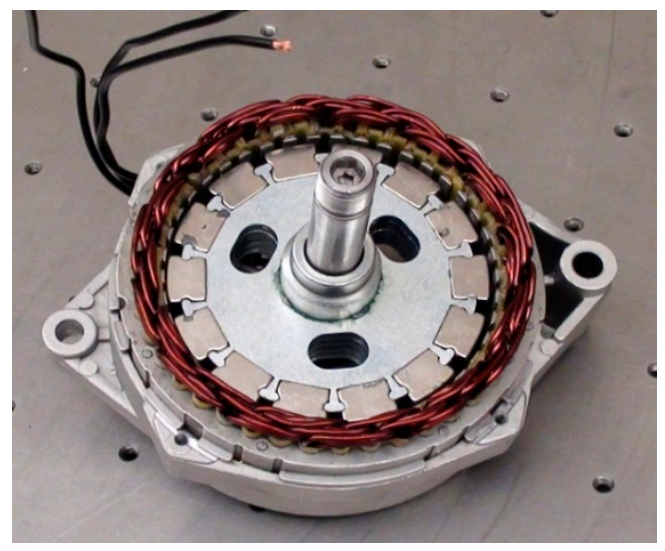

Figure 3. Demonstration machine: a three-phase permanent magnet generator.

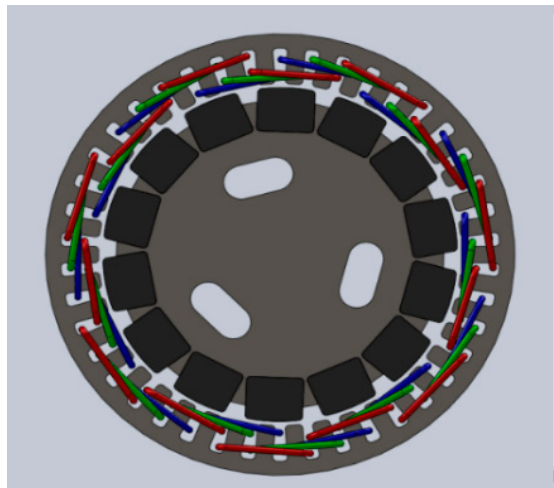

(a)

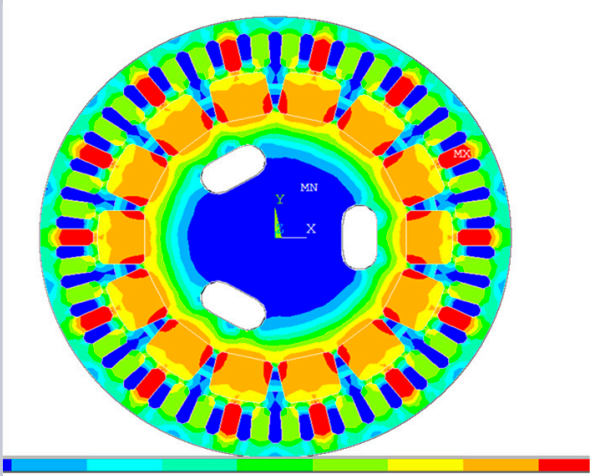

(b)

Figure 4. (a) CAD model of the demonstration machine. (b) FEA solution-flux density plot for the zero rotor angle.

Figure 2 compares the rotor velocity and stator current for one phase of a machine similar to the test machine, with cogging torque included and cogging torque ignored for two different input torques. The bottom plots are close-up views of the top plots. At higher velocities, the inertia of the rotor and load smoothened out the effect of the cogging torque. However, torque ripple (and the associated velocity ripple) was still present. Figure $2 \mathrm{~b}$ compares the rotor velocity for the same machine but with a smaller input torque. The effect of cogging torque was clearly more pronounced at low speeds and with a small torque input.

Limitations of the Classical Model. The main limitations of the classical model lie in how the model parameters are calculated and the assumptions made: (1) the flux linkage vs. the angle waveform is assumed to be perfectly sinusoidal (Equations (6) and (7)); (2) the stator inductance matrix is assumed to be constant (Equation (4)); (3) cogging torque is ignored; and (4) the flux linkage and stator inductance matrix are calculated analytically using expressions that do not account for material nonlinearities and hysteresis.

To address these limitations, the proposed hybrid model uses standard finite element software to more accurately estimate the model parameters that depend on the rotor angle, such as the flux linkage, stator inductances and cogging torque. The parameters are then incorporated in the dynamic equations of the classical model.

\subsubsection{Hybrid Dynamic Model of a Permanent Magnet Synchronous Generator}

The hybrid nonlinear state space model uses finite element analysis to obtain the rotor angle-dependent parameters, namely the flux linkage, stator inductance and cogging torque, while removing the limitations mentioned in Section 1.1.1. Unlike in [11], commercial finite element software (ANSYS) was used instead of custom code. ANSYS has built in 
functions for creating coils and calculating the inductance, flux linkage and co-energy, and it can handle nonlinear materials in addition to having a scripting language (APDL) which makes running multiple cases simple.

From the classical model, recognizing that both flux linkage due the PMs and the stator induction matrix are functions of the electrical angle, Equations (3) and (6) are changed to

$$
\begin{gathered}
\frac{d \vec{\psi}}{d t}=L_{s}(\theta) \frac{d \vec{i}}{d t}+\frac{d \psi_{m}(\theta)}{d \theta} \frac{d \theta}{d t} \\
\frac{\partial W_{c}}{\partial \theta}=\frac{1}{2} i^{T} \frac{d L_{s}(\theta)}{d \theta} \vec{i}+\overrightarrow{i^{T}} \frac{d \psi_{m}(\theta)}{d \theta}
\end{gathered}
$$

FEA provides discrete results at specific rotor angles to be used in conjunction with the dynamic model, which is continuous. FEA data must therefore be accessed in a way that makes it possible to incorporate them into the dynamic model; quantities such as the co-energy need to be differentiated with respect to the angle, which requires the FEA data to be differentiable.

Fourier Series Representation of Periodic Quantities. The periodic quantities obtained by FEA will be converted into a form useable by the continuous dynamic model by means of a Fourier series. The main advantage for using a Fourier representation is the possibility of taking derivatives analytically; thus, the pitfalls of using numerical schemes to estimate derivatives are avoided. Discrete quantities obtained by FEA analysis will be represented as a Fourier series of the following form:

$$
y(\theta)=a_{0}+\sum_{n}^{\infty} a_{n} \cos (n \theta)+b_{n} \sin (n \theta)
$$

where $a_{n}$ and $b_{n}$ are Fourier coefficients and $\theta$ is the electrical angle in radians. The Fourier coefficients are calculated off-line using the fast Fourier transform (FFT) function. This enables expression of the derivative with respect to the angle as

$$
\frac{d y(\theta)}{d \theta}=\sum_{n}^{\infty}-a_{n} n \sin (n \theta)+b_{n} n \cos (n \theta)
$$

\section{State Space Hybrid Dynamic Model.}

The proposed hybrid dynamic model that incorporates all the enhancements outlined above is arranged in a state space form, where all angular variables are electrical angles. The rotor and electrical angles and angular velocities are related by $\theta_{m}=P \theta / 2$ and $\omega_{m}=P \omega / 2$, where $\theta$ and $\omega$ are the electrical angle and angular velocity, respectively, $\theta_{m}$ and $\omega_{m}$ are the mechanical (rotor) angle and angular velocity, respectively, and $P$ is the number of poles in the machine. From Kirchhoff's and Newton's laws, the governing equations are

$$
\begin{gathered}
\vec{U}=-R_{s} \vec{i}+\frac{d \vec{\psi}}{d t} \\
T_{i n}+T_{\operatorname{cog}}-T_{e}-\frac{P}{2} B_{m} \omega=J \frac{P}{2} \frac{d \omega}{d t} \frac{\overrightarrow{d \psi}}{d t}=L_{s}(\theta) \frac{d \vec{i}}{d t}+\frac{d \overrightarrow{\psi_{m}}(\theta)}{d \theta} \frac{d \theta}{d t} \\
T_{e}=\frac{P}{2} \frac{\partial W_{c}}{\partial \theta}=\frac{P}{2}\left(\frac{1}{2} i^{T} \frac{d L_{s}(\theta)}{d \theta} \vec{i}+\overrightarrow{i^{T}} \frac{d \overrightarrow{\psi_{m}}(\theta)}{d \theta}\right)
\end{gathered}
$$

where Equation (11) is Kirchoff's voltage equation in the rotor circuit, Equation (12) is the torque equation in the rotor shaft and Equation (13) is the electrical torque equation. This defines the hybrid state space model: 


$$
\begin{aligned}
& \frac{d}{d t}\left[\begin{array}{c}
i_{a} \\
i_{b} \\
i_{c} \\
\omega \\
\theta
\end{array}\right]=\left[\begin{array}{ccc}
{\left[-R L_{s}(\theta)^{-1}\right.} & 0 & 0 \\
3 \times 3 & 0 & 0 \\
-\frac{P^{2}}{4 J}\left[\begin{array}{ccc}
i_{a} & i_{b} & i_{c}
\end{array}\right]\left[\frac{\partial L_{s}(\theta)}{\partial(\theta)}\right] & 0 & 0 \\
0 & 0 & 0
\end{array}\right]\left[\begin{array}{c}
i_{a} \\
i_{a} \\
i_{a} \\
\omega \\
\theta
\end{array}\right]+
\end{aligned}
$$

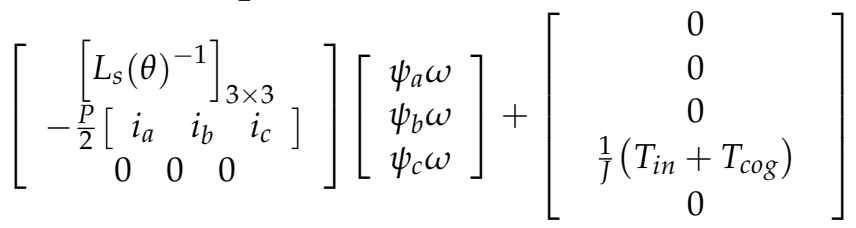

where $i_{a}, i_{b}, i_{c}, \omega$ and $\theta$ are the states of the system (currents of phases $a, b$ and $c$, the electrical angular velocity and the electrical angular position, respectively), $L_{s}(\theta)$ and the partial derivatives of $L_{S}(\theta)$ and $\Psi_{m}(\theta)$ with respect to $\theta$ are functions implemented as Fourier series from data extracted from FEA and $T_{i n}$ and $T_{\operatorname{cog}}$ are torques external to the model, which are treated as inputs. This formulation enables simple implementation in numerical environments such as Matlab and Simulink.

\section{Materials and Methods}

To demonstrate the validity and assess the performance of the proposed hybrid model, experimental measurements of the machine's performance were conducted on a threephase demonstration machine: the permanent magnet generator shown in Figure 3. The corresponding CAD model (Figure 4a) was used to import the geometry to FEA software (ANSYS), where the physical parameters of the machine could be calculated as a function of the rotor angle. Table 1 shows the physical characteristics of the demonstration machine.

Table 1. Physical parameters of the three-phase permanent magnet generator demonstration.

\begin{tabular}{lc}
\hline \multicolumn{1}{c}{ Dimension } & Value \\
\hline Rotor diameter & $0.1016 \mathrm{~m}$ \\
Stator inner diameter & $0.1049 \mathrm{~m}$ \\
Stator outer diameter & $0.1346 \mathrm{~m}$ \\
Number of stator slots & 42 \\
Number of permanent magnets & 14 \\
Number of stator phases & 3, delta connection \\
Air gap & $0.000254 \mathrm{~m}$ \\
Turns per phase & 3 \\
Stator winding pitch & Full \\
Cogging torque (peak) & $2.7681 \mathrm{~N}-\mathrm{m}$ \\
\hline
\end{tabular}

The cogging torque in the demonstration machine was significant, and it was measured as a function of the rotor angle using a mechanical torque wrench. Cogging torque measurements provided the opportunity to test the performance of the hybrid model while serving as measurable and verifiable parameters to ensure that the FEA simulations of the actual device were accurate.

Finite Element Model of the Demonstration Machine. Since the rotor had 14 permanent magnets, 180 electrical degrees corresponded to 25.714 mechanical degrees, and 52 simulations were performed for rotor angles ranging from 0 to 52 degrees. The ANSYS RACE macro was used to generate a series of racetrack coils arranged in a full-pitch threephase stator with three turns per phase, as could be found in the demonstration machine. The flux linkages and mutual and self-inductances of the stator coils were calculated for each degree of the rotor angle. The permanent magnet material used in the simulation was assumed to be $\mathrm{N}-42 \mathrm{HNdFeB}$, with a magnetic coercive force of $1.35 \times 106 \mathrm{~A} /$ Turns and a magnetic remanence of $1.3 \mathrm{~T}$ (MatWeb) [18]. The iron parts of the machine were 
assumed to be 1020 steel alloy, whose hysteresis curve was obtained from ANSYS's internal material library.

Flux linkage was calculated using an analytical procedure (Equation (6)); the FEA and analytical flux linkage are compared in Figure 5a. The analytical expression resulted in a peak flux linkage of $0.028 \mathrm{~Wb}$, while the FEA gave a peak of $0.025 \mathrm{~Wb}$, a $10 \%$ difference. The FEA considers effects such as hysteresis, saturation and material nonlinearity, resulting in a more accurate estimation of flux linkage. Physical parameters extracted from the FEA are calculated offline and need only be estimated once. Mutual inductance and self inductance obtained from the FEA simulations are shown in Figure 6 for each phase. Each plot shows the corresponding mutual and self-inductances after fitting the values calculated via FEA using Fourier series of the electrical angle $\theta$.

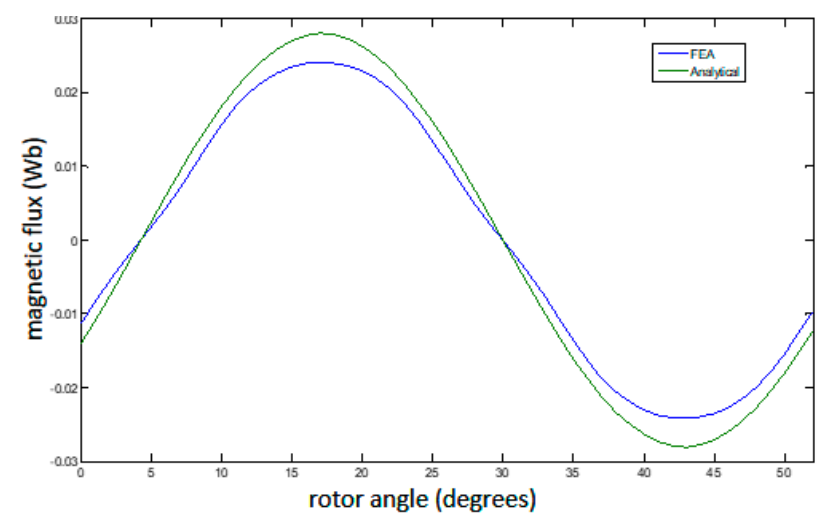

(a)

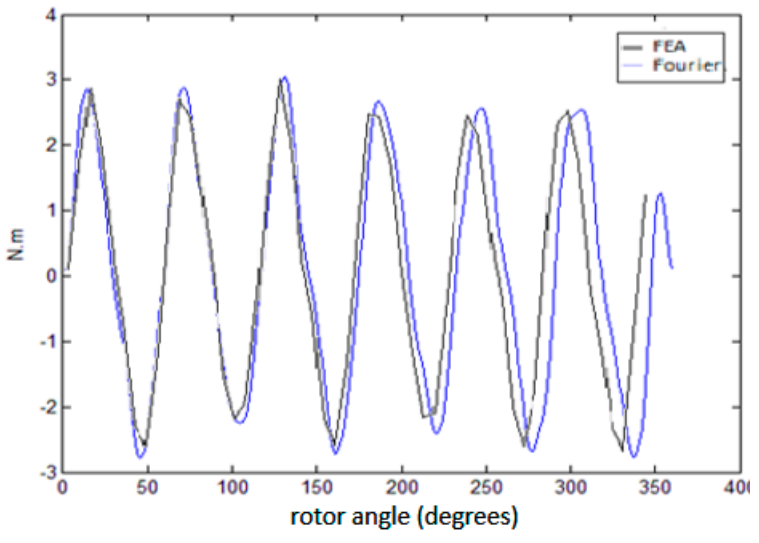

(b)

Figure 5. (a) Flux linkage: comparison of FEA and analytical flux linkage as a function of the rotor angle. (b) Cogging torque: comparison of FEA and Fourier representation.
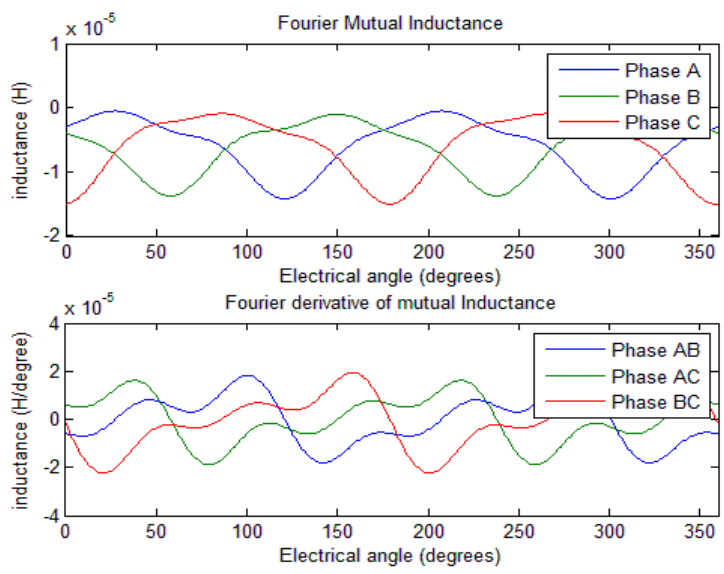

(a)
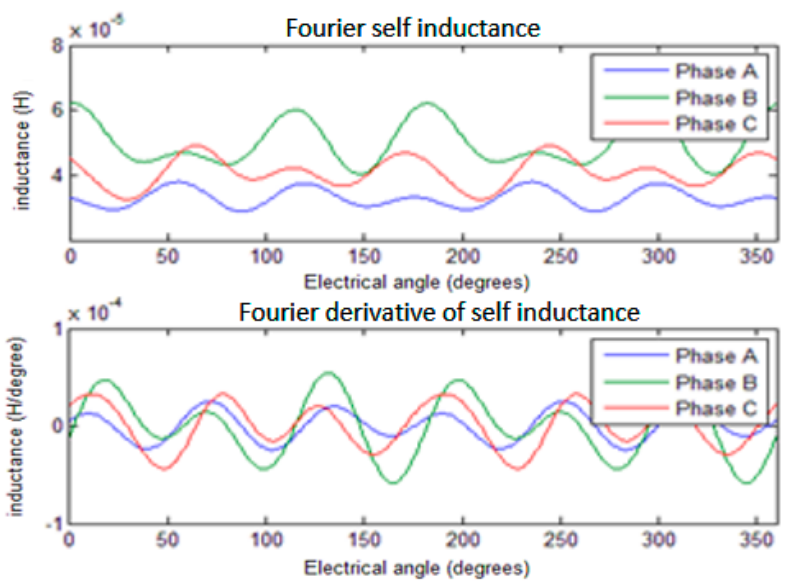

(b)

Figure 6. Fourier representations and their derivatives relative to the rotor angle: (a) mutual inductance and (b) self inductance.

The Fourier coefficients for the physical machine parameters (mutual and self-inductance, cogging torque and the corresponding derivatives relative to the rotor angle) were obtained and implemented as Matlab functions of the rotor angle [19]. The cogging torque was also obtained via FEA, and it too was represented by a Fourier series function, as shown in Figure 5b. The classical model provides no means for calculating cogging torque. 
Experimental Set-up: Dynamic Response Experiments. To measure the dynamic and steady state responses of the demonstration machine, the stator currents and rotor angular velocity must be measured while the machine is being driven by a prime mover, or in this case, a drive motor having a belt transmission with a 1.6 torque ratio, as shown in Figure 7a. The generator was connected to a three-phase "wye"-connected resistive load consisting of three 0.35 -ohm power resistors. The stator currents were measured using F.W. Bell NT-15 current sensors in series with the load, which produced a voltage proportional to the current. The drive motor was a Baldor SD25-30-A1 driven by an AMC servo controller, which was commanded via analog voltage. The current supplied to the drive motor was recorded using the same current sensors mentioned above; the motor current was used to determine the torque applied by the drive motor, with a torque constant of $0.41 \mathrm{Nm} / \mathrm{A}$ and a rotor inertia of $0.0158 \mathrm{~kg} / \mathrm{m}^{2}$.

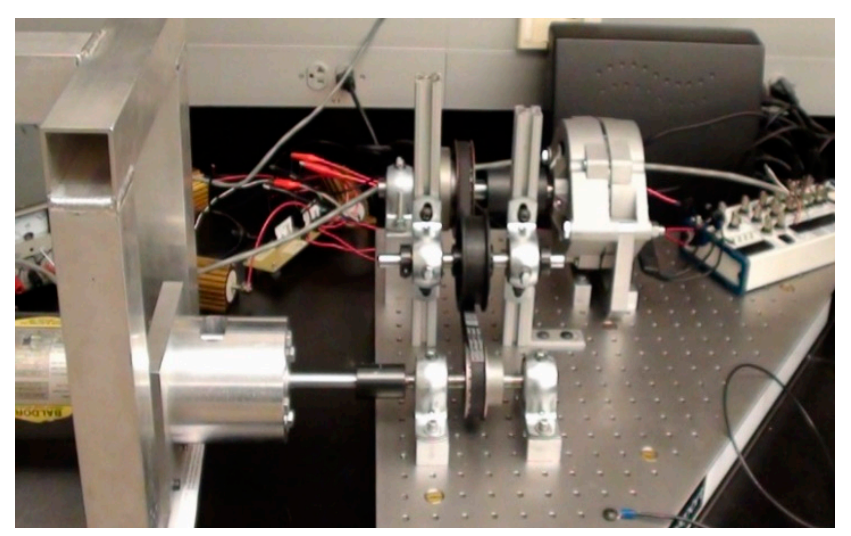

(a)

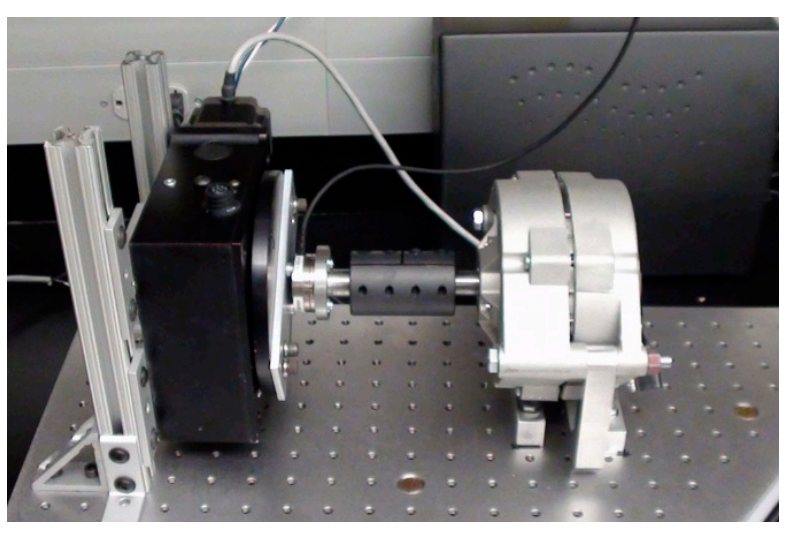

(b)

Figure 7. Experimental set-up (a) for dynamic measurements and (b) to measure the angular dependent parameters.

The angular velocity was measured using a quadrature optical encoder connected to the generator rotor. The encoder output was converted to a voltage signal via a US-Digital ETACH2 analog tachometer. A National Instruments PXI DAQ system was used both data acquisition, data capture and control of the AMC driver. Using this set-up, three types of experiments were performed.

Transient Dynamics. To measure the transient response of the machine, the drive motor was started from zero RPM, and the generator was allowed to accelerate all the way to a steady state and settle while the stator currents, rotor velocity and drive motor current were acquired. The current drawn by the drive motor (which was proportional to the input torque) became the input torque to the hybrid model. Two types of transient experiments were performed: a full-speed test, where the drive motor was allowed to run up to the rated current limit of the motor drive driver and power supply, and a low-speed test, where the input torque was limited, resulting in a slower response.

Steady State Characteristics. The steady state characteristics of the demonstration machine were measured after the rotor velocity attained a steady state. At this point, the input current to the drive motor could be adjusted to a nearly constant value, and the data were recorded. The steady input conditions were then applied to the model as a constant input torque. Both the full-speed and low-speed steady state experiments were performed.

Short Circuit Dynamics. The short circuit dynamics test is a test of an electrical machine's behavior during a sudden short circuit condition during operation. The machine's dynamic behavior during this condition (rotor speed and stator currents) is vital to the design of robust electrical machines that are able to withstand hazardous conditions. For the demonstration machine, a sudden short circuit condition was implemented by adding a switch that short circuited the stator windings. Under such conditions, the stator currents, rotor velocity and drive motor current (i.e., with torque applied) were measured. 
Angular Parameter Measurements. The objective of this set of experiments was the determination of the cogging torque and phase inductances of the demonstration machine as function of the rotor angles. Due to the high cogging torque, rotating and holding the rotor at a desired angle was difficult. This was overcome by using a stepper motor in combination with a worm gear drive connected to the rotor, as shown in Figure $7 \mathrm{~b}$.

Cogging Torque. An ATI-IA 6-axis force-torque transducer was installed between the stepper motor and test machine to measure the cogging torque as the stepper moved the rotor at small angular increments. A LabVIEW program was written to handle both the stepper motor control and acquisition of the data in the PXI DAQ.

Measuring Stator Inductances. To measure the line-to-line inductances of the demonstration machine as a function of the rotor angle, the measurement set-up included the stepper motor drive connected to the test machine, as shown in Figure $7 \mathrm{~b}$. The line-to-line inductances were measured using a MCP BR2822 LCR meter. This meter measured the inductance, capacitance and resistance by forming an LCR circuit with the component being measured and sending a periodic voltage signal with a user-selectable frequency. The inductance was measured from 0 to 26 mechanical degrees, which corresponded to the 180 electrical degrees of the demonstration machine.

\section{Results and Discussion}

\subsection{Transient Measurements and Simulation Results}

The model required an estimation of the moment of inertia and the viscous damping coefficient. The moment of inertia for the drive motor was found in its documentation, and the moment of inertia for the drive system was estimated from the dimensions and weight of the components. To estimate the viscous damping, the test machine was disconnected from its load (which resulted in no stator currents and thus no electromagnetic reaction torque), effectively decoupling the electrical and mechanical systems. The experimental set-up was driven in this configuration, and the rotor angular velocity and the input torque were recorded. A separate dynamic simulation of the mechanical system was only run using the input torque, and the viscous damping coefficient was adjusted until the velocity response matched the experimental values. The input torque applied by the drive motor was recorded as the current supplied to the motor, and the captured torque waveform was applied to the simulation. The input torque waveforms (Figures 8 and 9a) were applied to both the classical and hybrid model simulations, and the results are shown in Figures 8 and $9 \mathrm{~b}$.
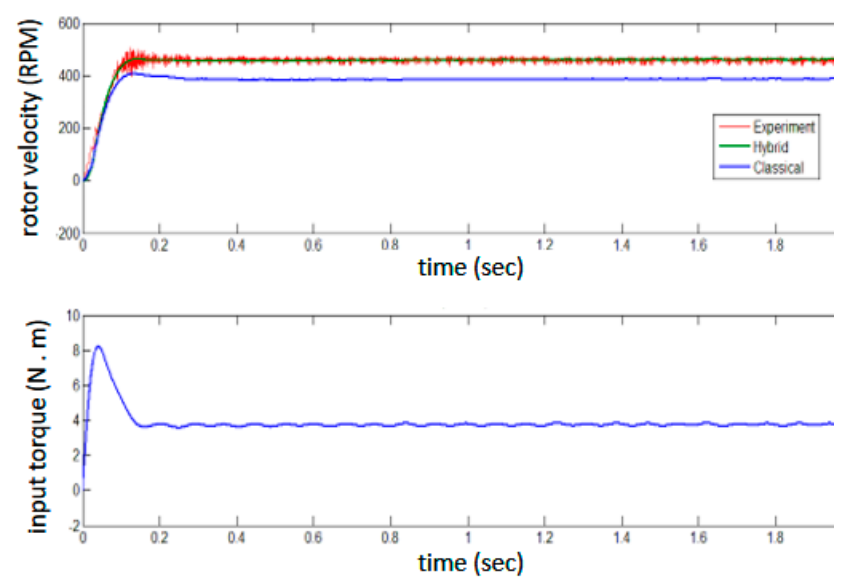

(a)

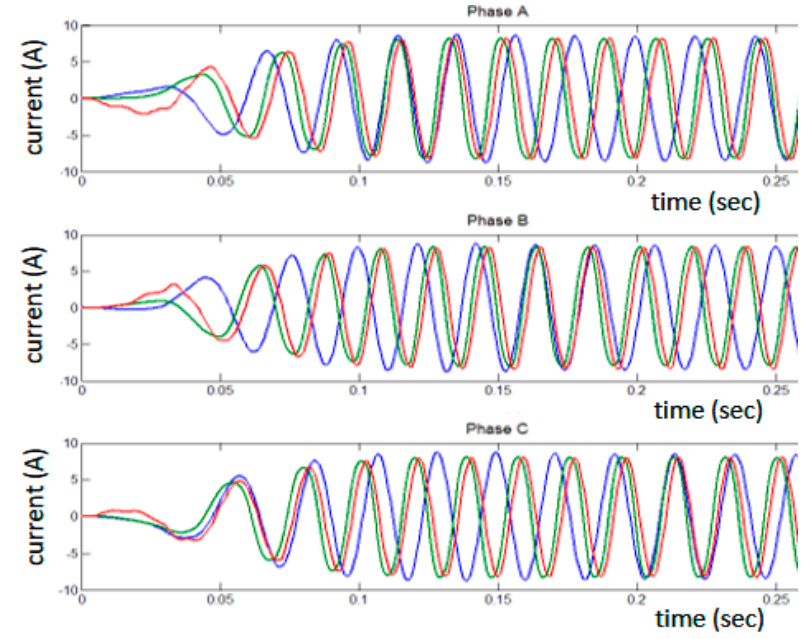

(b)

Figure 8. Full speed transient response, showing (a) the rotor velocity and input torque and (b) a comparison of the stator currents. The plots show a comparison of the classical model (blue trace), hybrid model (green) and experimental measurements (red). 

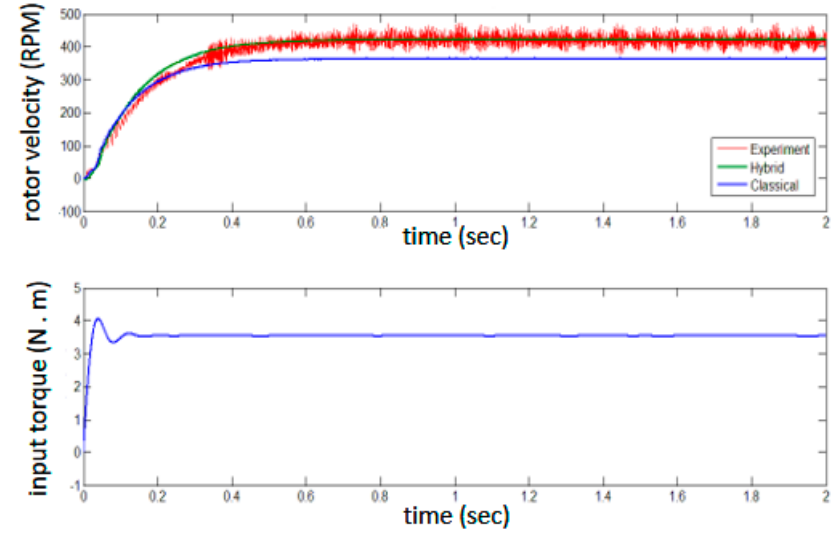

(a)
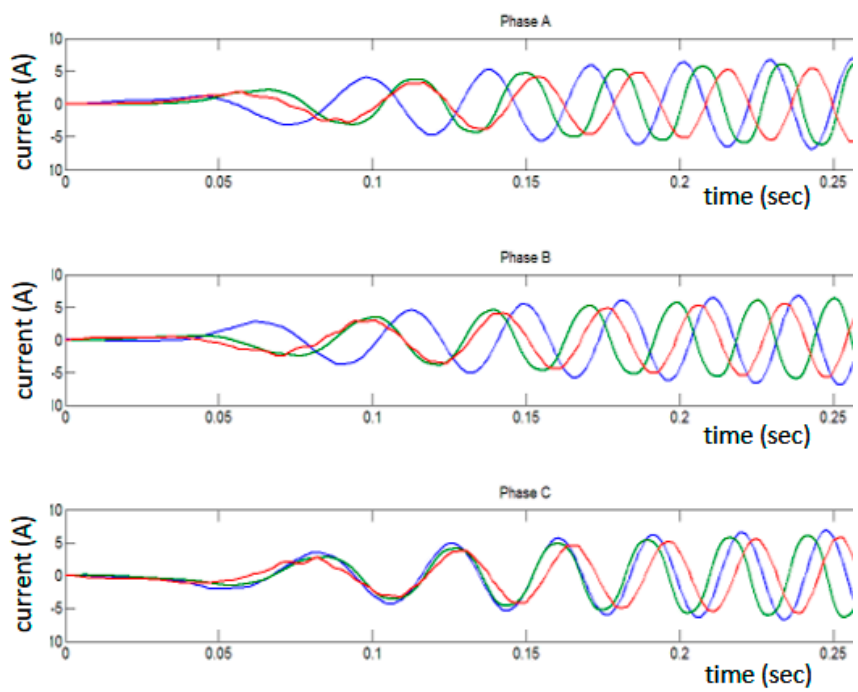

(b)

Figure 9. Low-speed transient response, showing (a) the rotor velocity and input torque and (b) a comparison of the stator currents. The plots show a comparison of the classical model (blue trace), hybrid model (green) and experimental measurements (red).

Figure 8 shows good agreement between the hybrid model rotor velocity estimate and the measured velocity. The classical model rotor velocity was lower than the measurement. This was mainly due to the analytical flux linkage value being greater than the FEA value. The greater flux linkage resulted in higher stator currents, which in turn exerted more EM torque, resulting in a lower rotor velocity for the same torque input. The good match between the hybrid model and the measured velocity shows that the FEA flux linkage was indeed closer to the real value and demonstrates the benefits of including the FEA results in the model. The noise observed on the measured velocity curve was introduced by the timing belt system.

Figure $8 \mathrm{~b}$ shows the stator currents for each phase for the full-speed transient test, in which the measured currents are compared to both the classical model and the hybrid model. The hybrid model showed good agreement with the measured data, while the classical model differed in the peak current and frequency, due to both the increased flux linkage in the classical model and the frequency mismatch given by the lower estimate of the rotor velocity.

Figure 9 shows results of the low-speed transient test. The noise present in the measured velocity signal was due to the dynamics of the timing belt system. Once again, the hybrid model and experimental results had good agreement, while the classic model's velocity estimate was lower than the measured and hybrid models due to the same reasons mentioned for the full-speed case. In the prediction of the stator currents, the hybrid model agreed well with the measured data, with the classical model showing higher peak currents and a lower frequency than the measurements, as was mentioned in the full-speed case.

\subsection{Steady State Measurements and Simulation Results}

To compare the results quantitatively, the root mean square of the stator currents was calculated, and it was used as the metric for direct comparison. For the rotor velocities, the mean was calculated. The measured velocity curves in Figure 10 (full speed) and Figure 11 (low speed) show spurious frequencies, as does the input torque applied by the drive motor. These were due to the dynamics of the timing belt system and cogging torque. However, in both the full-speed and low-speed cases, the hybrid model was capable of correctly matching the measured angular velocity values. The average torque applied by the drive motor was input into the hybrid and classical models as a constant torque. The 
average velocities of the experimental data and model results showed a discrepancy of $15.0 \%$ in the classical model, while the discrepancy for the hybrid model was only $2.18 \%$.

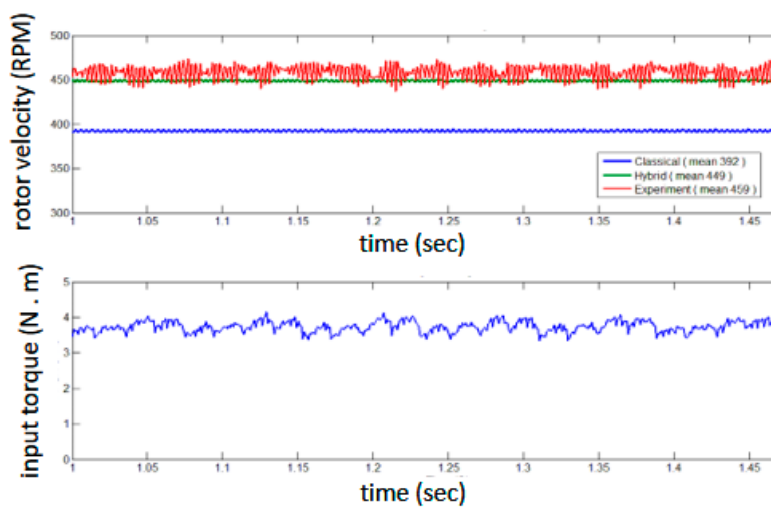

(a)
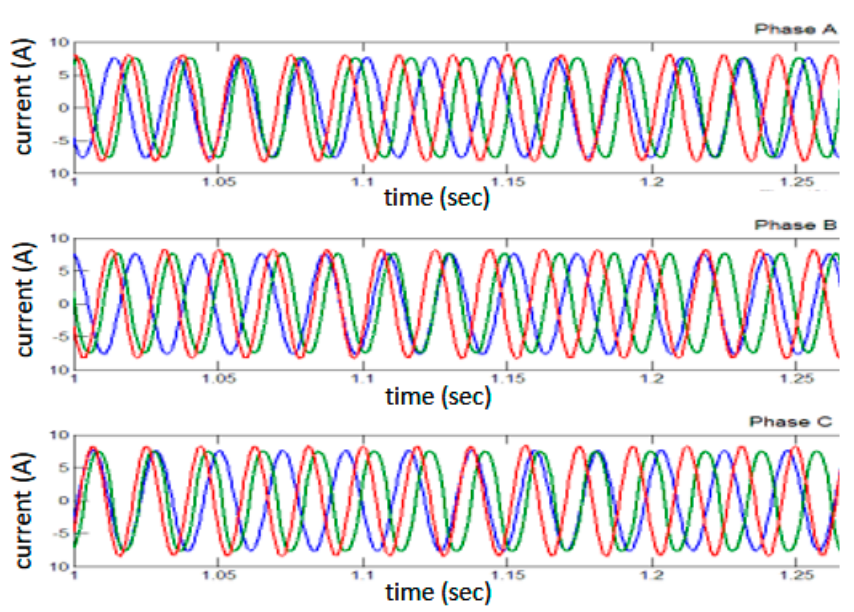

(b)

Figure 10. Full-speed steady state response, showing (a) the rotor velocity and input torque and (b) a comparison of the stator currents. The plots show a comparison of the classical model (blue trace), hybrid model (green) and experimental measurements (red).
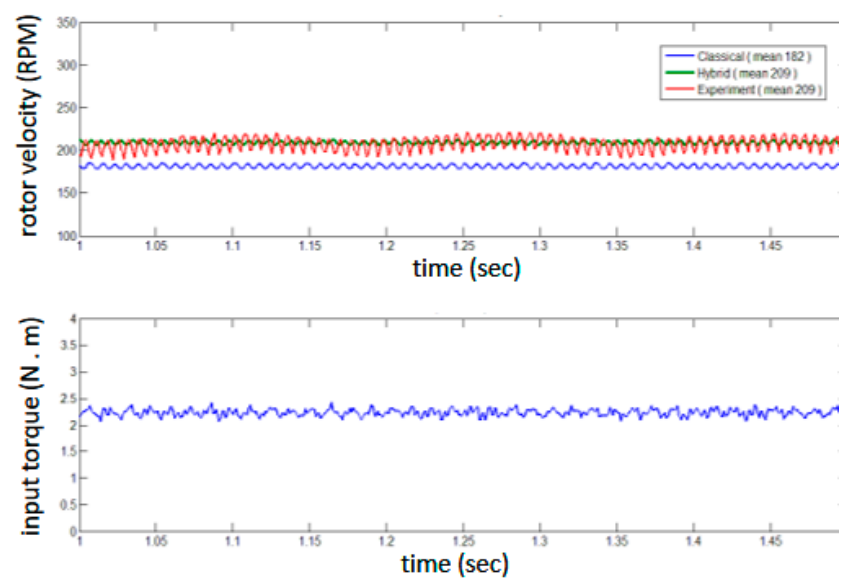

(a)
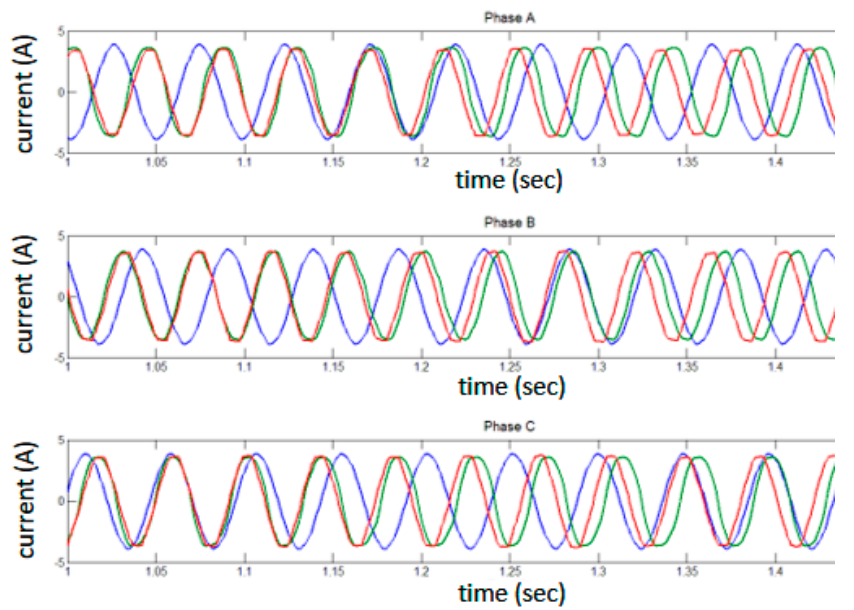

(b)

Figure 11. Low-speed steady state response, showing (a) the rotor velocity and input torque and (b) a comparison of the stator currents. The plots show a comparison of the classical model (blue trace), hybrid model (green) and experimental measurements (red).

The rotor velocity predicted by the classical model was considerably lower than the measurement due to the increased EM torque caused by the increased stator currents, as was mentioned before.

The RMS values of the stator currents in the three phases of the test machine showed a 5\% difference. This was because the test machine was not electrically balanced (i.e., the inductances and resistances of each phase were not identical). The frequencies of the three waveforms did not match exactly, which was due to differences in the rotor angular velocity in each case. The hybrid model showed a closer match with the measurements since it provided better velocity estimation. The rotor velocity predicted by the classical model was smaller than both the hybrid model and the experimental data, and the waveforms had correspondingly lower frequencies. The error between the estimated and measured 
rotor velocities was $2.4 \%$ for the hybrid model and $14 \%$ for the classic model. The reason for this, again, was the analytical flux linkage value used in the classical model.

\subsection{Short Circuit Measurements and Simulation Results: Hybrid Model}

Figure 12 shows the results for the short circuit test. The sudden increase in the stator current resulted in a sudden increase in the electromagnetic torque, which caused the machine to slow down abruptly. The simulated and experimental short circuit dynamics were in good agreement for the hybrid model. The ripple observed in both results was due to the cogging torque, as its effect was more pronounced at low speeds. The additional frequency contents in the measured velocity were due to the effect of the timing belt dynamics.

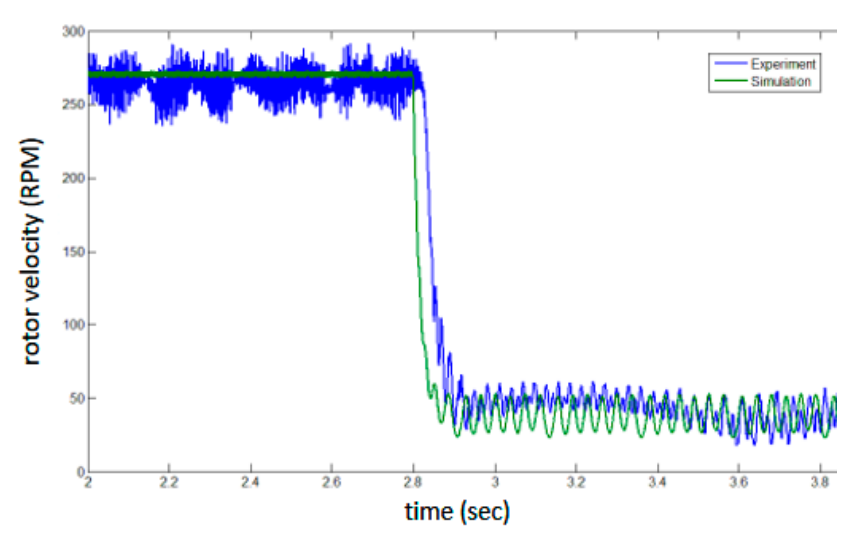

(a)

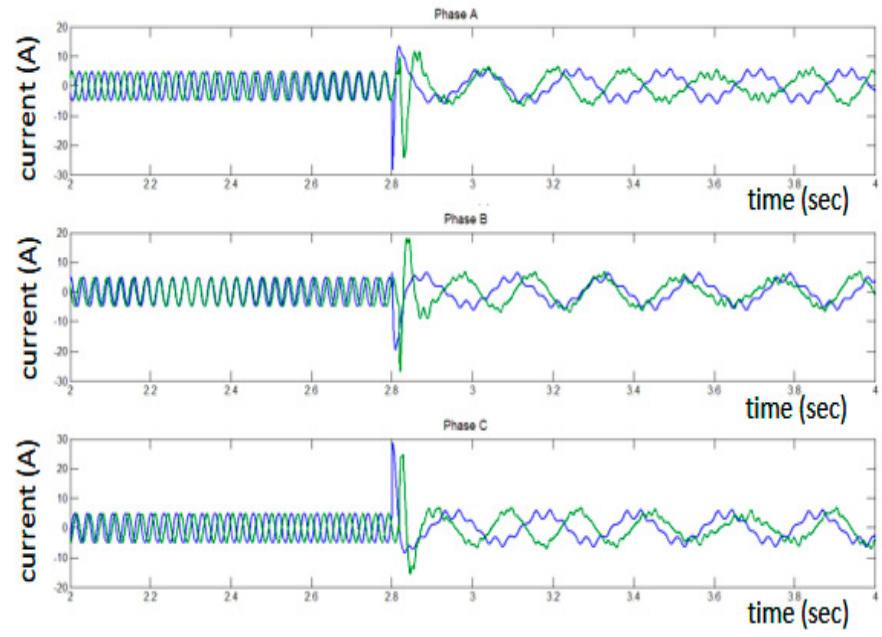

(b)

Figure 12. Short circuit test response. (a) Comparison of the rotor velocities. (b) Comparison of the stator currents. The plots show a comparison of the hybrid model (green trace) and the experimental measurements (blue).

Figure $12 \mathrm{~b}$ shows the stator currents that corresponded to the short circuit test. When the short occurs, a sudden spike in stator current is clearly visible for all three phases, and the peak current was about three times the current prior to the short. After the short occurred, the machine settled into a new steady state with a greatly reduced frequency due to the reduced rotor velocity. The effect of the cogging torque in Figure $12 \mathrm{~b}$ is also apparent. Note that the hybrid model was able to model these effects with great detail.

Angular Dependent Parameters. The measured angular parameters were compared to the results obtained from the FEA simulation, and the results are shown in Figure 13. The parameters measured were the cogging torque and line-to-line stator inductances.

Cogging Torque. The experimental and FEA results for the cogging torque are compared in Figure 13a. While the peak values showed very good agreement (3\% error), the mismatch in waveform shapes was due to the torque sensor slipping while the rotor was being turned.

Line to Line Inductances. There was no agreement between the FEA results and measurements of the line-to-line inductances. The stator windings for each phase only had three turns, resulting in very low inductance and leading to numerical errors in the FEA calculation. Even with significant errors in inductance, the model was able to archive good agreement with the experimental data due to the fact that in a PM machine, the flux linked by the magnets was significantly larger than the flux linked by the mutual inductance of the stator coils. This also highlights the importance of flux linkage in the dynamics of a PM electrical machine. 


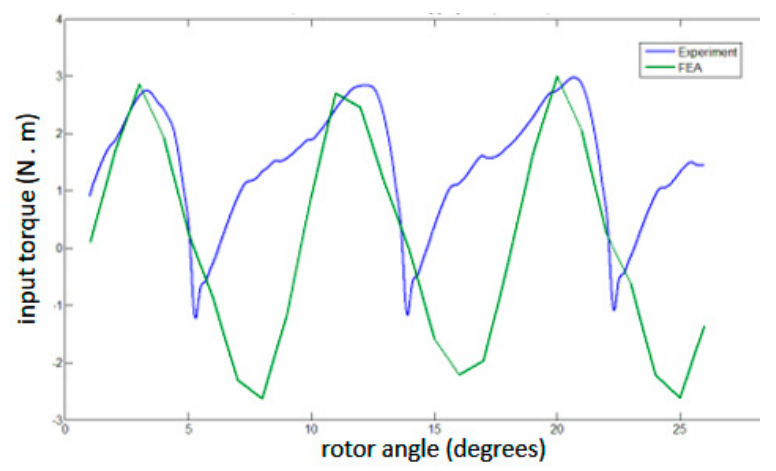

(a)

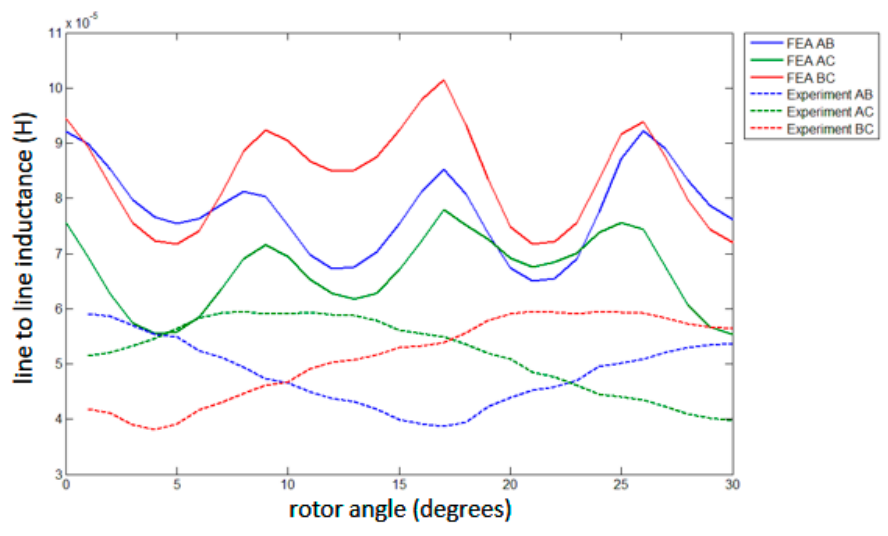

(b)

Figure 13. Comparison of angular dependent parameters for experimental measurements vs. FEA predictions. (a) Cogging torque. (b) Line to line inductances.

\section{Conclusions}

The hybrid dynamic state space model presented here was able to accurately predict (within a 7\% difference) the electrical and mechanical dynamics of a three-phase permanent magnet generator. The model was able to predict the magnitude and frequency of the stator currents as well as the stator current waveforms, and it incorporated the cogging torque. These features address many of the limitations of the classical model by using FEA to determine the rotor angle-dependent parameters, namely the flux linkage, stator inductances and cogging torque. The proposed hybrid model was validated against experimental data, and its performance was compared with that of the classical model for permanent magnet generators.

The hybrid model could perform better if other unmodeled dynamic effects could be included, such as the timing belt spring constant, viscous damping in the bearings and others. Numerical errors in the FEA calculation of the stator inductances also had an effect on the overall fitness of the hybrid model. In all, the hybrid model reduced error prediction by $15 \%$ compared with the classical model.

The use of FEA to obtain the angular dependent parameters is not without its disadvantages, as evidenced by the mismatch between the FEA-calculated inductances and the measured values. The main contributions of FEA rely on its ability to calculate the angular flux linkage due to the permanent magnets and its ability to handle nonlinear materials and complex geometries. Flux linkage is the single most important factor that determines the magnitude and shape of the stator current waveforms and electromagnetic torque. Thus, the advantages offered by FEA in calculating not only the magnitude but also the waveform shape of the flux linkage cannot be understated.

Cogging torque is the second most significant effect on the dynamics of the PM generator. FEA is able to model cogging torque accurately, and FEA can be used to test strategies for mitigating cogging torque during the design stage. The effect of cogging torque is less pronounced at higher speeds, but with today's emphasis on low-RPM, high-torque machines, cogging torque has become an important design and modeling consideration.

Author Contributions: H.M.G.: conceptualization, methodology, project administration, review and editing; T.F.: software development, experimental validation, formal analysis and original draft preparation; Q.Z.: investigation and literature review. All authors have read and agreed to the published version of the manuscript.

Funding: This research was partly supported by the U.S. National Science Foundation through GOALI Award ECCS-1028621.

Conflicts of Interest: The authors declare no conflict of interest. 


\section{References}

1. Boldea, I.; Nasar, S.A. Linear Electric actuators and Generators. IEEE Trans. Energy Convers. 1999, 14, 712-717. [CrossRef]

2. Norhisam, M.; Norafiza, M.; Sia, C.Y. Double Stator Type Permanent Magnet Generator. In Proceedings of the 2009 Students Conference on Research and Development, Serdang, Malaysia, 16-18 November 2009.

3. Chai, F.; Cui, S.; Cheng, S. Study of a Novel Double-stator Permanent-Magnet Electrical Machine. In Proceedings of the IEEE International Electric Machines and Drives Conference, Madison, WI, USA, 15 July 2003; pp. 1375-1378.

4. Chai, F.; Xia, J.; Gong, H.; Guo, B.; Cheng, S. Torque Analysis of a Double-Stator Permanent Magnet Synchronous machine for Hybrid Electric Vehicle. In Proceedings of the IEEE Vehicle Power and Propulsion Conference, Harbin, China, 3-5 September 2008.

5. Zhang, D.; Niu, S.; Chau, K.T.; Jiang, J.Z.; Gong, Y. Design and Analysis of a Double-Stator Cup-Rotor Directly Driven Permanent Magnet Wind Power Generator. In Proceedings of the CES/IEEE 5th annual Power Electronics and Motion Control. Conference, Shanghai, China, 14-16 August 2006; pp. 1-5.

6. Vas, P. Vector Control of AC Machines. Monographs in Electrical and Electronic Engineering 22; Oxford Science Publications: Oxford, UK, 1990.

7. Levi, E.; Levi, V.A. Impact of Dynamic Cross-Saturation on Accuracy of Saturated Synchronous Machine Models. IEEE Trans. Energy Convers. 2000, 15, 224-230. [CrossRef]

8. Melkebeek, J.A.; Willems, J.L. Reciprocity Relations for the Mutual Inductances Between Saturated Salient-Pole Machines. IEEE Trans. Ind. Appl. 1990, 26, 107-114. [CrossRef]

9. Iglesias, I.; Garcia-Tabarnes, L.; Tamarit, J. A d-q Model for the Self-Commutated Synchronous Machine Considering the Effects of Magnetic Saturation. IEEE Trans. Energy Convers. 1992, 7, 768-776. [CrossRef]

10. El-Serafi, A.; Kar, N.C. Methods for Determining the Intermediate-Axis Saturation Characteristics of Salient-Pole Synchronous Machines from the Measured D-Axis Characteristics. IEEE Trans. Energy Convers. 2005, 20, 88-97. [CrossRef]

11. Poltschak, F.; Amrhein, W. A dynamic nonlinear model for permanent magnet synchronous machines. IEEE Int. Symp. Ind. Electron. 2008, 724-729. [CrossRef]

12. Lyshevski, S.E. Electromechanical Systems, Electric Machines and Applied Mechatronics; CRC Press: New York, NY, USA, 1999.

13. Guru, B.S.; Hiziroglu, H.R. Electric Machinery and Transformers; Oxford University Press: New York, NY, USA, 2001.

14. Lovatt, H.C.; Watterson, P.A. Energy Stored in Permanent Magnets. IEEE Trans. Magn. 1999, 35, 505-507. [CrossRef]

15. Dosiek, L.; Pillay, P. Cogging Torque Reduction in Permanent Magnet Machines. IEEE Trans. Ind. Appl. 2007, 43, 1565-1571. [CrossRef]

16. Zhu, Z.; Howe, D. Influence of Design Parameters on Cogging Torque in Permanent Magnet Machines. IEEE Trans. Energy Convers. 2000, 15, 407-412. [CrossRef]

17. Hossain, S.; Deshpande, U. Modeling of Practical Non-Idealities in a Permanent Magnet Synchronous Motor Drive. In Proceedings of the IEEE Industry Applications Conference, Salt Lake City, UT, USA, 12-16 October 2003; pp. 1941-1945.

18. MatWeb. Alliance N-42H Neodymium Iron Boron Magnetic Material. Available online: www.matweb.com (accessed on 4 January 2014).

19. Zhu, Z.Q. A Simple Method for Measuring Cogging Torque in Permanent Magnet Machines. In Proceedings of the IEEE Power \& Energy Society General Meeting, Calgary, AB, Canada, 26-30 July 2009.

20. Meeker, D. Magnetic Circuit Derivation of Energy Stored in a Permanent Magnet, April 2007. Available online: https://www. femm.info/wiki/pmenergy (accessed on 4 January 2014). 